\title{
Evaluación del programa de formación en investigación 2006- 2008, de la Facultad de Cultura Física, Deporte y Recreación de la USTA*
}

\section{Evaluation of Training in Research 2006-2008, of the Faculty of Physical Culture, Sports and Recreation USTA}

Asceneth María Sastre Cifuentes**

Recibido: 16 de diciembre de 2009

Revisado: 22 de enero de 2010 Aceptado: 18 de febrero de 2010

\section{Resumen}

Este proyecto evaluó el programa de formación en investigación vigente con un enfoque mixto, que vinculó a toda la comunidad académica mediante procesos de muestreo estratificado y recogió la experiencia registrada en documentos durante los últimos 2 años. Se aplicaron técnicas análisis descriptivo y de análisis de contenido de tipo categorial. Se reconocieron como aspectos positivos los elementos conceptuales, los contenidos de formación y el Ejercicio de Investigación Semestral (EIS) como estrategia pedagógica del

* Esta investigación fue desarrollada en el marco de las acciones propias de la Coordinación del Programa de Formación en Investigación de la Facultad de Cultura Física, Deporte y Recreación de la Universidad Santo Tomás, entre febrero de 2008 y marzo de 2009, en la línea de investigación de Pedagogía de la cultura física del grupo Cuerpo, Sujeto y Educación, categorizado D por Colciencias.

** La autora es psicóloga egresada de la Universidad de los Andes. Magíster en Psicología Clínica y de Familia, creadora y docente investigadora del programa de Formación en Investigación de la Facultad de Cultura Física, Deporte y Recreación de la USTA, cuyos procesos lideró desde la fundación de la Facultad en 1998 y hasta 2009. Actualmente ejerce como docente investigadora en esta Facultad y es líder del grupo Cuerpo Sujeto y Educación, categorizado D por Colciencias. Correo electrónico: mariasastre@usantotomas.edu.co 
programa mientras que se identificó la necesidad de mejoramiento en los aspectos de gestión, especialmente en el tiempo destinado a los contenidos y actividades de formación, al equipo docente y a la estructura administrativa que lo sostiene. El estudio permitió el planteamiento de soluciones a las dificultades del programa que conllevaron una propuesta de actualización la cual logró ser aprobada por las autoridades académicas de la Universidad, y que actualmente está en proceso de implementación.

Palabras clave: formación en investigación.

\section{Abstract}

This project evaluated the current Research Training Program, using a mixed approach. It worked on the whole academic community by means of a stratified sampling procedure and the registered experience in documents during the two last years. The quantitative data were analyzed by means of descriptive statistics and the qualitative ones by means of category contents analysis. The information shows as positive aspects the conceptual elements as well as the themes, contents and the Semester Research Exercise (SRE) as a the pedagogic strategy of the program. It shows, moreover that the management aspects require changes especially in the time destined to contents and training activities, the professors team and the administrative structure which supports it. This study identific of the program deficiencies as well as its benefits, and the emerging solutions that effectively involued a transformation proposal in the general program which today, after its approval by the University academic authorities, is under execution.

Key words: research training. 


\section{Introducción}

Desde el 2001 la Facultad de Cultura Física, Deporte y Recreación, mediante su programa de formación en investigación se ha propuesto promover el desarrollo de actitudes, intereses y habilidades investigativas, superando el énfasis en el aprendizaje de contenidos que ha caracterizado la formación en investigación, tradicionalmente incorporada en los currículos, al fomentar en el estudiante la reflexión y la participación activa y permanente en la construcción de conocimientos, la recuperación del interés por la comprensión de la realidad, la lectura, la observación, una postura interrogativa y reflexiva, la interpretación, la argumentación y la socialización del conocimiento, acciones que lo sitúan en una postura crítica, afectiva y ética desde la cual pueda vincularse en la propuesta de soluciones y alternativas contextualizadas a la realidad estudiada.

Este programa es la respuesta a la oferta de formación de la USTA que enuncia textualmente que:

El profesional en Cultura Física, Deporte y Recreación es un profesional preparado para hacer lecturas comprensivas de contextos, que le permitan transformar la realidad social y personal $[\ldots]$ a través de procesos de investigación y desarrollo del pensamiento crítico, para la construcción permanente de alternativas que generen bienestar y mejor calidad de vida (USTA, 2009).

El proceso de formación encaminado a este propósito, sujeto a modificaciones que respondan a las necesidades sentidas por la comunidad académica, debe someterse a un proceso de evaluación permanente que garantice su pertinencia. Habiendo transcurrido dos años desde el último ajuste, resulta no solo oportuna una evaluación de los efectos generados en el mejoramiento de los procesos y los productos de la formación en investigación, sino necesaria de cara a los procesos de registro calificado y acreditación de alta calidad en que la facultad viene comprometiéndose desde el 2005. Adicionalmente, el compromiso de la USTA con la instauración de 
un sistema de gestión de calidad conforme a las normas ISO 9001 y 2000, implica desarrollar rigurosos procesos de seguimiento, evaluación y mejoramiento de los procesos de formación profesional.

En el marco del actual currículo, la última reforma al programa de formación se llevó a cabo en julio de 2006, cuando los resultados de la evaluación desarrollada hicieron evidente la necesidad de introducir reformas con base en la identificación de puntos críticos. Así pues, urge volver sobre la experiencia para revisar de qué modo se están logrando los propósitos de la formación en investigación para, en consecuencia, articular los espacios académicos y sus contenidos, las prácticas investigativas y todo el movimiento investigador de la facultad y diseñar planes de mejoramiento, desde los resultados de una evaluación sistemática de las experiencias y productos de la formación investigativa en la Facultad. Este proyecto tiene el valor de retroalimentar el pensum, orientar decisiones acerca de los énfasis en la formación, las necesidades prioritarias de formación en el campo, las metodologías y las posibles modificaciones que garanticen la validez y pertinencia del currículo en el campo de la investigación, aportando además de manera significativa a los procesos de autoevaluación que caracterizan un programa de calidad, justamente por tener el fundamento necesario para la propuesta y ejecución de planes de mejoramiento continuo, que hoy en día son requisito sine qua non del registro calificado, la acreditación de alta calidad y los certificados de calidad ICONTEC con que está comprometida la Universidad.

En consecuencia, se propone evaluar el programa actual de formación en investigación a partir de las apreciaciones de la comunidad académica en torno a sus contenidos, estrategias pedagógicas, organización administrativa, equipo docente y productos, para lo cual se compromete con las siguientes preguntas de investigación: ¿responde el actual programa a los objetivos de la formación en investigación?, ¿las modificaciones realizadas se han constituido efectivamente en mejoras en el proceso de formación?, ¿qué aspectos críticos surgen como foco de atención para eventuales propuestas de mejoramiento?, ¿en qué dirección deben proponerse cambios en 


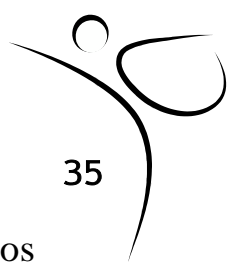

el programa actual de modo que mejore la calidad de los procesos de formación en investigación?

Este proyecto se plateó como objetivo general evaluar el programa de formación en investigación desarrollado en los últimos 2 años, y específicamente identificar la percepción que del programa de formación tienen los estudiantes y docentes vinculados al proceso en sus aspectos organizativos, en los alcances de los contenidos de formación, en los procesos de evaluación, los productos, el equipo de docentes de investigación, las dimensiones éticas implicadas en el proceso, la participación de los coordinadores de líneas y el valor de la estrategia pedagógica empleada (Ejercicio de Investigación Semestral - EIS) en la formación de los estudiantes como investigadores. En segundo término, identificar el nivel de logro en el desarrollo de las competencias en cada nivel de formación, las necesidades de mejoramiento en función de la jornada y el nivel de formación de los estudiantes y describir la funcionalidad de la estructura administrativa que sostiene el proceso de cara al logro de los objetivos de formación.

\section{Metodología}

Se empleó una metodología mixta que recogió las apreciaciones de los actores del proceso, docentes y estudiantes a partir del uso tanto de técnicas cuantitativas (cuestionario de pregunta cerrada), cualitativas (grupos de discusión, observaciones escritas, actas, testimonios). Es un estudio de tipo evaluativo, pues confronta los datos de la realidad con unas condiciones esperadas o deseables que son justamente las que propone el programa de formación en sus principios, objetivos, contenidos y estrategias pedagógicas, y que son el referente de la evaluación. La población corresponde a la conformada por 590 estudiantes de la Facultad de Cultura Física, matriculados en el período 2008-1, de las jornadas diurna y mixta, y de primero a octavo semestre, según la estadística de Matriculados por Facultad y género para el período académico 
2008-1 del Sistema académico de la Universidad, y 23 docentes vinculados al programa de formación en Investigación durante el período 2008-1 como docentes del campo de la investigación y como coordinadores de líneas. Para la recolección de la información cuantitativa se realizó un muestreo estratificado, buscando una representación estadística de las dos jornadas y los ocho niveles de formación en cada una de ellas. La muestra definitiva fue de 207 estudiantes y 14 docentes.

La información cualitativa se obtuvo de una muestra documental por accesibilidad y conveniencia conceptual (2006-2008), de registros escritos (actas) de las reuniones de cierre y planeación del campo de la investigación de cada semestre académico desde 2006-2 hasta 2008-1, de observaciones escritas de estudiantes y docentes en los cuestionarios aplicados, y del testimonio de la coordinación del programa en su experiencia durante los años considerados en el estudio.

Se construyeron dos cuestionarios de pregunta cerrada. El cuestionario A orientado a la medición de los logros (en porcentaje de estudiantes que los alcanzan) según apreciación de los docentes de investigación, considerando los desempeños mínimos esperados para cada nivel. La puntuación que arroja es en términos de porcentaje de logros.

El cuestionario B se orientó a recoger las apreciaciones acerca del programa de formación en investigación, en 58 ítems agrupados en ocho subescalas: aspectos organizativos, alcances de los contenidos de formación, procesos de evaluación, productos, dimensión ética, equipo docente, coordinadores de líneas, Valor de la estrategia pedagógica - EIS).

Otras técnicas de recolección de información incluyeron la consulta de archivos, actas, testimonios escritos de los actores y descripciones cualitativas anexas a los cuestionarios aplicados. 


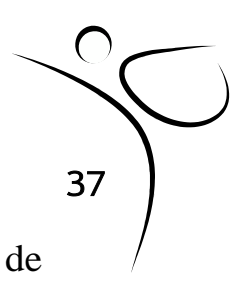

Como técnicas de análisis de la información, se aplicó un análisis de frecuencias (estadística descriptiva) para la información cuantitativa, y un análisis de contenido de tipo categorial para la información cualitativa.

\section{Resultados}

Respecto a la percepción actual del programa de formación por parte de estudiantes y docentes vinculados al proceso, la información cuantitativa deja ver cómo la mayor parte de las respuestas son casi siempre y siempre, en porcentajes que en conjunto oscilan entre el $50 \%$ y el $75 \%$, con una cierta desventaja de la mixta en la aplicación de programa de formación (promedio inferior en la evaluación realizada por estudiantes de la jornada mixta, excepto en los ítems 4,5, 10, 16, 23, 34, 39, 44 y 45, en que los puntajes de la jornada mixta superan ligeramente los de la diurna).

Se aprecian necesidades inmediatas de ajuste y mejoramiento en las dos jornadas, prestando especial atención a los aspectos en los que converge una pobre evaluación: tiempo destinado a las asesorías, el póster como estrategia de aprendizaje, las estrategias didácticas utilizadas por los docentes en sus clases, los espacios de discusión entre docentes de investigación y coordinadores de líneas, y los pósteres de los coordinadores de línea como modelo para el aprendizaje de la estrategia.

Atendiendo a los puntajes arrojados en cada subescala, se evidencia que los aspectos del programa mejor evaluados son, en su orden: el valor del EIS como estrategia de aprendizaje, los productos del programa de formación y los contenidos del programa, los aspectos éticos, los docentes, los aspectos organizativos, los coordinadores de líneas y a evaluación. 
La comparación entre la evaluación hecha por docentes y estudiantes evidencia una evaluación ligeramente más positiva por parte de los estudiantes pero ambos reconocen el valor del EIS como estrategia pedagógica, y de los contenidos de formación. Se evidencia una evaluación pobre especialmente en la participación de los coordinadores de líneas, la organización y la evaluación, y un puntaje que no satisface lo esperado en lo relativo al desempeño docente.

Se juzga como bueno el valor del programa de formación en investigación, su aporte en el aprendizaje y la formación profesional, el desarrollo de habilidades, y el EIS como una estrategia útil de cara al logro de las metas. Como aceptables se consideran el alcance de los contenidos de formación, los principios, objetivos y pertinencia del programa. Tanto docentes como estudiantes coinciden en considerar deficiente el tiempo destinado a la formación, a las asesorías y a la discusión interna entre docentes, el destino de los productos de la formación, el perfil de los docentes (competencias y ajuste a lineamientos) y el sistema de evaluación. El uso de la estrategia del póster debe recibir ajustes. Se considera insuficiente la difusión de los productos de investigación de los estudiantes, el sistema de evaluación, y la participación y responsabilidad de los coordinadores de líneas en los procesos de orientación de los productos y su difusión.

El nivel de logro en el desarrollo de las competencias en cada nivel de formación es bajo, según la apreciación de los docentes responsables del proceso, tanto como el nivel de desarrollo de competencias investigativas semestre a semestre.

Por su parte, la información cualitativa permite comprender en profundidad lo que subyace a estas apreciaciones, al dejar ver los argumentos que respaldan los juicios evidenciados por la evaluación cuantitativa: 
Tabla 1.

\begin{tabular}{|c|c|c|}
\hline $\begin{array}{l}\text { Categoría } \\
\text { analítica }\end{array}$ & Docentes & Estudiantes \\
\hline $\begin{array}{l}\text { Requerimientos } \\
\text { frente a proceso } \\
\text { de evaluación }\end{array}$ & $\begin{array}{l}\text { Acoger seriamente como cri- } \\
\text { terios los desempeños estable- } \\
\text { cidos por nivel y articularlos } \\
\text { semestre a semestre. } \\
\text { Unidad de criterios en orien- } \\
\text { tación y evaluación del EIS. } \\
\text { Tiempos comunes para el } \\
\text { acompañamiento a docentes, } \\
\text { concertación de metodo- } \\
\text { logías y procedimientos de } \\
\text { evaluación. } \\
\text { Evaluación y rediseño de las } \\
\text { formas de evaluación de los } \\
\text { desempeños en cada nivel. }\end{array}$ & $\begin{array}{l}\text { Fortalecer el sentido del EIS } \\
\text { desde el espacio académico. } \\
\text { Planear estrictamente los } \\
\text { tiempos, de acuerdo con las } \\
\text { metas por semestre. } \\
\text { Ampliar el tiempo para la } \\
\text { construcción del EIS. } \\
\text { Permitir el trabajo individual. } \\
\text { Exigir presencia en la } \\
\text { socialización. } \\
\text { Individualizar la evaluación } \\
\text { considerando esfuerzo y } \\
\text { responsabilidad de cada quien. } \\
\text { Replantear los objetivos, } \\
\text { momento de presentación, } \\
\text { aspectos logísticos y } \\
\text { destino de los pósteres de } \\
\text { investigación. }\end{array}$ \\
\hline $\begin{array}{l}\text { Requerimientos } \\
\text { frente a acom- } \\
\text { pañamiento a } \\
\text { estudiantes }\end{array}$ & $\begin{array}{l}\text { Tiempo para asesorar a los } \\
\text { estudiantes. } \\
\text { Revisar responsabilidad docen- } \\
\text { te en la actitud del estudiante } \\
\text { hacia la sustentación del EIS. } \\
\text { Estrechar acompañamiento de } \\
\text { alto nivel de exigencia. }\end{array}$ & $\begin{array}{l}\text { Ampliar tiempos para el } \\
\text { desarrollo de los contenidos } \\
\text { del Programa (clase). } \\
\text { Ampliar tiempos para } \\
\text { asesoría del EIS: estrechar } \\
\text { acompañamiento en } \\
\text { construcción del EIS. }\end{array}$ \\
\hline
\end{tabular}




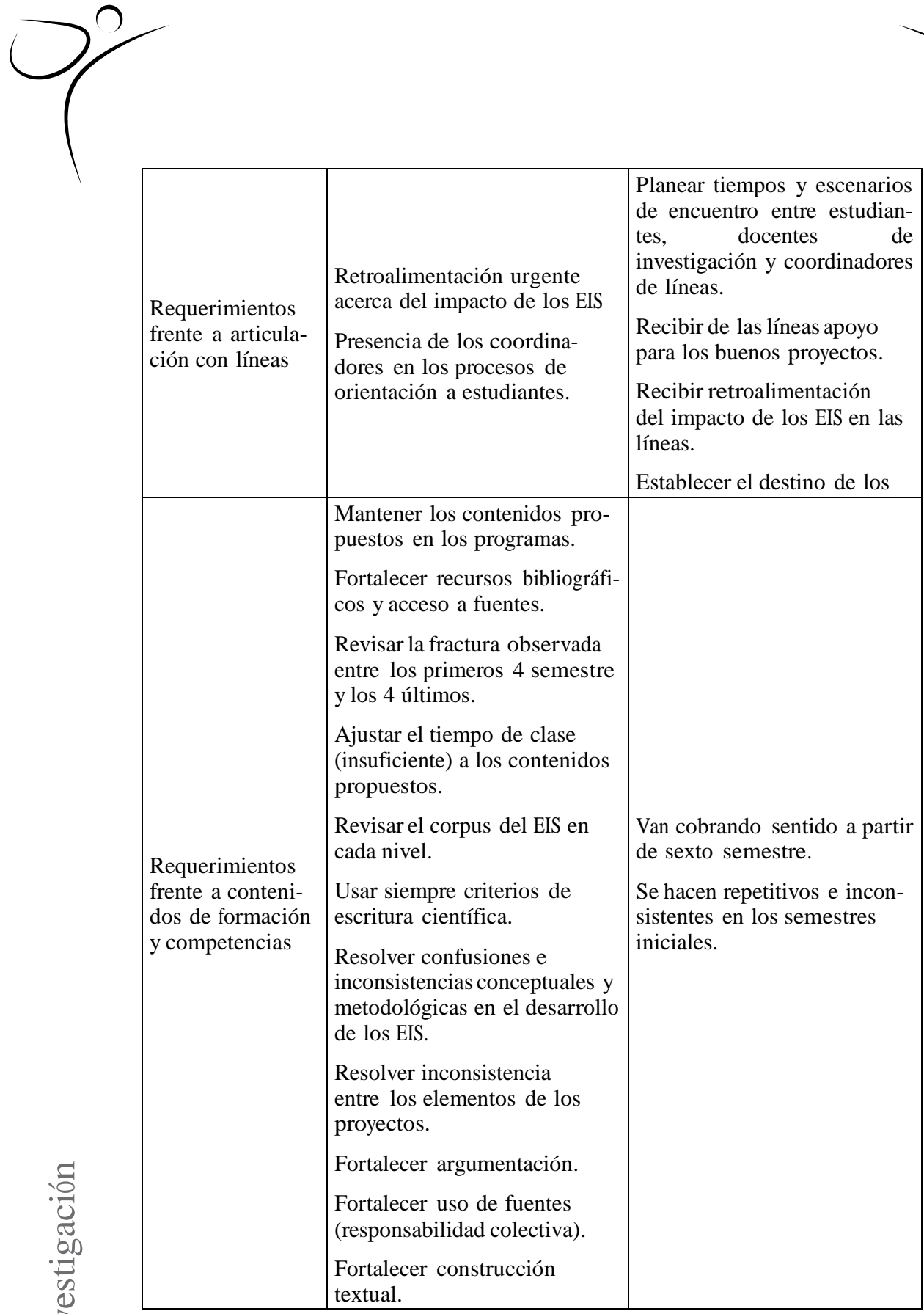


Requerimientos frente a formación y desempeño docente

Aspectos de gestión del programa
Mejorar nivel de apropiación de programas y pedagogías apropiadas.

Unificación en la ejecución de programas entre docentes del mismo nivel.

Docentes con formación en investigación, compromiso y tiempo.

Enfatizar claridad conceptual, claridad metodológica.

Enfatizar didácticas apropiadas.

Enfatizar criterios y formas de evaluación.
Unidad conceptual y procedimental entre docentes del mismo nivel y entre niveles consecutivos.

Capacitación docente (conocimientos en investigación y habilidades para enseñar).

Selección docente conforme a su idoneidad: unificar perfil docente por lo alto.

Evitar improvisaciones en la construcción del EIS.

Mejorar la exigencia.

Diseñar e informar cronogramas oportunamente.

Revisar las metodologías y didácticas empleadas.

Reconocer el interés/desinterés del docente como fuente del interés/desinterés del estudiante por la investigación.

Diferenciar y respetar los tiempos destinados a clase y a asesorías.

Mantener las reuniones informativas iniciales con docentes, procesos de inscripción, registro y reporte de resultados EIS, difusión de documentos orientadores, disponibilidad de la coordinación (comunicación abierta) y bases de datos.

Fortalecer reuniones docentes, aspectos logísticos, de evaluación y destino de los pósteres, mecanismos de información y comunicación (carteleras) y procesos de registro e informes de progreso de estudiantes. 


\begin{tabular}{|c|c|}
\hline $\begin{array}{l}\text { Aspectos } \\
\text { valorados en } \\
\text { el docente de } \\
\text { investigación }\end{array}$ & $\begin{array}{l}\text { Conocimiento y experiencia } \\
\text { en investigación. } \\
\text { Claridad conceptual y } \\
\text { metodológica. } \\
\text { Uso de métodos } \\
\text { participativos. } \\
\text { Interés por el aprendizaje } \\
\text { del estudiante y esfuerzo por } \\
\text { facilitarlo (dedicación). } \\
\text { Entusiasmo con los conteni- } \\
\text { dos que enseña. } \\
\text { Preparación. } \\
\text { Retroalimentación oportuna } \\
\text { y orientadora. } \\
\text { Exigencia. } \\
\text { Puntualidad. } \\
\text { Organización y orden con } \\
\text { tiempos, trabajos, evaluacio- } \\
\text { nes y notas. }\end{array}$ \\
\hline $\begin{array}{l}\text { Aspectos } \\
\text { valorados en } \\
\text { el docente de } \\
\text { investigación }\end{array}$ & $\begin{array}{l}\text { Coherencia. } \\
\text { Diferenciación y respeto a los } \\
\text { tiempos de clase/asesoría. } \\
\text { Equitativo. } \\
\text { Dominio de la información } \\
\text { (documentos guía, fechas, } \\
\text { actividades y procedimientos). } \\
\text { Trato respetuoso. } \\
\text { Reconocimiento y valoración } \\
\text { del esfuerzo. }\end{array}$ \\
\hline
\end{tabular}


La información recogida permite afirmar, en términos generales, que el programa tiene un buen nivel de aceptación por parte de los estudiantes. Sin embargo, aquellos ítems que recibieron más respuestas que expresan deficiencias en el programa, o inconsistencias en la experiencia desde la cual los estudiantes lo evalúan, señalan posibilidades de mejoramiento.

Por ejemplo, los juicios acerca de la forma de evaluar el tiempo destinado en el plan de estudios a la formación en investigación, el póster como estrategia que favorece el desarrollo de habilidades en investigación y la formación y competencias del equipo de docentes de investigación, parecen desprenderse de la experiencia individual de los estudiantes en el programa, que liga con los docentes que de manera particular lo han acompañado en su proceso, y que lo han hecho de manera más o menos idónea, o comprometida. Así, la calidad del proceso de formación evidentemente está mediada por la calidad de los docentes y por el rigor con que desempeñan sus funciones.

Algo similar ocurre con la evaluación que hacen respecto al conocimiento que los estudiantes tienen de las guías, lineamientos, reglamentos e indicadores que regulan el proceso de formación en investigación, que algunos dicen no conocer y otros dicen hacerlo casi siempre. Nuevamente, las inconsistencias en la forma de respuesta sugieren inconsistencias en la aplicación del programa por parte de los docentes, que es necesario entrar a analizar, y que pueden dejar ver deficiencias en su formación, en su motivación y compromiso, o en las condiciones en que deben desarrollar sus funciones docentes (tiempo, recursos, etc.). 
Cuando se interpretan los datos arrojados por la comparación entre las dos jornadas, se evidencia una cierta desventaja de la mixta en la aplicación del programa de formación, lo que confirma en todas y cada una de las subescalas. Es necesario prestar más atención a la forma en la que el programa se aplica en la jornada de la noche, especialmente, atendiendo a la coordinación y acompañamiento a los docentes a cargo del mismo, que usualmente están desarticulados de los procesos de seguimiento implementados con mas rigor en la jornada diurna, por disponibilidades horarias de la coordinación.

Surge como necesidad inmediata de mejoramiento el tiempo destinado a asesorías, la utilización del póster como una verdadera estrategia de aprendizaje, las estrategias didácticas implementadas por los docentes en sus clases, los espacios de discusión entre docentes de investigación y coordinadores de líneas, y los pósteres de los coordinadores de línea como modelo para el aprendizaje de la estrategia.

Es evidente, por otra parte, que lo que el programa plantea y se propone (el EIS como estrategia de aprendizaje, los productos generados por el programa y los contenidos abordados), se reconoce significativo y de valor para la formación por parte de la comunidad académica, así como los aspectos éticos implicados en ella. Los docentes ejecutores del programa, encargados de llevar a la realidad las propuestas y planes; sin embargo, son regularmente evaluados, así como los aspectos organizativos, lo que entre otras cosas incluye en organización entre los coordinadores de líneas y los procesos de evaluación. De ello se puede concluir que el programa tiene efectivamente fortalezas en sus aspectos conceptuales, las cuales son reconocidas por la comunidad académica, pero en su puesta en marcha presenta falencias que no permiten el logro cabal de sus objetivos: es necesario hacer los ajustes en aspectos referidos a recursos (humanos, de tiempo) y procedimientos.

La apreciación del porcentaje de estudiantes que alcanzan los logros correspondientes a cada nivel tiende a ser mejor en la jornada 


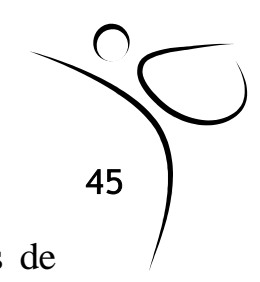

diurna, pero en ningún caso superan el $65 \%$ de los estudiantes de cada nivel, y en la mayoría de los casos es inferior al 50\%. Aunque se reconoce que esta medida resultó de una apreciación hecha por los docentes de investigación y no directamente del desempeño de los estudiantes conforme a los logros mínimos establecidos para cada nivel, da cuenta de la necesidad de ajustar las metodologías empleadas en la formación, (y con esto los tiempos y recursos necesarios) de tal forma que garantice el desarrollo de las habilidades que el programa se propone, en la mayoría de los estudiantes.

En cuanto al nivel de desarrollo de competencias investigativas, semestre a semestre, los resultados señalan, no sólo porcentajes de logros insuficientes nivel a nivel, sino el modo en que, a pesar de no alcanzar los logros de niveles inmediatamente anteriores, los estudiantes son promovidos al siguiente nivel, con carencias y sensibles vacíos en su formación. Esta es por supuesto una responsabilidad del docente, que compromete sus procedimientos para fortalecer el aprendizaje y para evaluar el modo en que los estudiantes alcanzan los desempeños esperados. Así, un programa que conceptualmente se reconoce como valioso, pero que operativamente no logra ajustarse para cumplir sus propósitos, no podrá alcanzar sus objetivos a menos que se ajuste la logística que le da vida y que de manera sensible compromete el desempeño de los docentes que tienen bajo su responsabilidad el desarrollo del mismo.

Cabe señalar que los estudiantes tienden a ser más benévolos en la evaluación que los docentes, lo que señala un nivel de expectativas más alto por parte de estos últimos y grado importante de consciencia y autocrítica frente al proceso, que se constituye ciertamente en un recurso para mejorar pues las deficiencias observadas no parecen asociarse a ingenuidad o quizás a la irresponsabilidad de los docentes en el ejercicio de sus funciones, sino que sugiere que existen condiciones desfavorables que pueden dar cuenta de tales deficiencias. El análisis de estas condiciones lo provee la información cualitativa en este trabajo. 
El análisis de las convergencias entre los principales actores de la comunidad académica, docentes y estudiantes, ofrece importantes orientaciones y señala la urgencia de introducir modificaciones básicamente en los aspectos operativos que no logran facilitar la puesta en marcha de un programa que conceptualmente plantea un proceso de formación de calidad, pero que requiere de unos procesos de gestión y toma de decisiones que correspondan a sus propósitos. Más que ajustar los propósitos y contenidos del programa a las condiciones de logística y operativización disponibles, se trata de ajustar estas últimas a las necesidades de un proceso de formación adecuadamente concebido y proyectado.

Como investigación formativa que se articula a la investigación propiamente dicha en la Facultad, es urgente fortalecer los vínculos entre una y otra en la persona de los coordinadores de línea que tendrían que ejercer no sólo como investigadores sino como facilitadores de los procesos formativos y de retroalimentación acerca del impacto de los EIS en las líneas de investigación: su aporte y sentido. Por tanto, se reclama la presencia de los coordinadores en los procesos de orientación a estudiantes en espacios de discusión, encuentro, asesoría u orientación, así como mejores procesos de difusión de la información acerca del trabajo en las líneas. Los estudiantes requieren de las líneas de apoyo para los buenos proyectos, es decir, que se recojan los productos y se anime su proyección desde el trabajo de los estudiantes.

Respecto a la formación de los docentes es clara la necesidad de seleccionar para el campo docentes con formación en investigación, que puedan asumir cabalmente el compromiso de formar en ello y que dispongan del tiempo necesario para hacerlo. Se debe enfatizar en la claridad conceptual, y en la claridad metodológica requerida en un docente, para asumir la formación de otros y no generar confusiones, así como en la importancia de desarrollar las didácticas apropiadas, que faciliten el aprendizaje y desarrollo de competencias en investigación y estén en condiciones de manejar criterios y formas de evaluación adecuadas. 


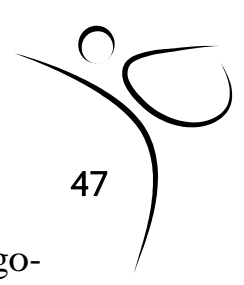

Un aporte fundamental de la información cualitativa, en una categoría que emergió a partir de los comentarios acerca del desempeño de sus docentes (ver resultados), son los criterios con los que evalúan a un buen/mal profesor, que pueden constituirse en elementos para la evaluación y selección de docentes.

\section{Conclusiones}

Aunque se reconocen aspectos positivos en el programa (especialmente de orden conceptual), es en su puesta en marcha, referida a los aspectos de gestión donde se requiere de manera urgente hacer ajustes especialmente en el tiempo destinado a los contenidos y actividades de formación, el equipo docente y la estructura administrativa que sostiene el programa. En concreto se concluye que frente a las dificultades identificadas, pueden proponerse alternativas de solución, como se muestra a continuación: 
Tabla 2.

\begin{tabular}{|c|c|}
\hline Dificultades identificadas & Propuestas \\
\hline $\begin{array}{l}\text { Tiempo de clase excesivamente limitado } \\
\text { para cubrir los contenidos de formación. }\end{array}$ & $\begin{array}{l}\text { Aumentar el tiempo de clase por lo } \\
\text { menos en la mitad del actual, y dife- } \\
\text { renciarlo claramente de los espacios } \\
\text { para asesoría individual. }\end{array}$ \\
\hline $\begin{array}{l}\text { Número de créditos que apenas si permite } \\
\text { el desarrollo de la clase en su dimensión } \\
\text { presencial y en nada la calidad en los pro- } \\
\text { cesos de asesoría ni el trabajo indepen- } \\
\text { diente del estudiante. }\end{array}$ & $\begin{array}{l}\text { Aumentar el número de créditos en } \\
\text { razón del tiempo requerido para el } \\
\text { desarrollo de los contenidos pro- } \\
\text { pios de cada nivel de formación, } \\
\text { tanto como de la necesidad de ase- } \\
\text { soramiento individual y del trabajo } \\
\text { independiente. }\end{array}$ \\
\hline $\begin{array}{l}\text { Inadecuada distribución del tiempo desti- } \\
\text { nado a los espacios académicos, con refe- } \\
\text { rencia a los énfasis que requiere la forma- } \\
\text { ción en sus diferentes etapas o ciclos. }\end{array}$ & $\begin{array}{l}\text { Fusionar en único espacio acadé- } \\
\text { mico los tres de habilidades inves- } \\
\text { tigativas, y en uno solo diseño me- } \\
\text { todológico, ampliando en cada caso } \\
\text { el número total de créditos, con la } \\
\text { consecuente ampliación de las horas } \\
\text { de clase y de trabajo independiente. }\end{array}$ \\
\hline $\begin{array}{l}\text { La secuencia rígida de ocho espacios aca- } \\
\text { démicos no permite la flexibilidad curricu- } \\
\text { lar y retrasa a los estudiantes al condicio- } \\
\text { nar el progreso en la secuencia, semestre } \\
\text { a semestre. }\end{array}$ & $\begin{array}{l}\text { Estadística puede tomarse más tem- } \\
\text { pranamente en la formación y no } \\
\text { estar condicionada por pre-requi- } \\
\text { sito diferente al espacio académi- } \\
\text { co de Introducción al pensamiento } \\
\text { científico. } \\
\text { Puede tomarse antes, después o al } \\
\text { tiempo con habilidades investiga- } \\
\text { tivas y proyecto de investigación, } \\
\text { y definitivamente antes de diseño } \\
\text { metodológico. }\end{array}$ \\
\hline $\begin{array}{l}\text { Insuficiente tiempo para asesorar la cons- } \\
\text { trucción de los EIS en forma adecuada y } \\
\text { satisfactoria. }\end{array}$ & $\begin{array}{l}\text { Contar con al menos media hora } \\
\text { de asesoría por subgrupo, cada tres } \\
\text { semanas, mejoraría sustancialmente } \\
\text { la calidad del proceso de asesoría } \\
\text { que en la actualidad muestra ser } \\
\text { deficiente. }\end{array}$ \\
\hline
\end{tabular}


Insuficiente tiempo del docente (tres horas) para ofrecer al trabajo de los estudiantes el seguimiento (lectura, revisión y corrección de sus productos) necesario para el desarrollo de las competencias propuestas por el plan de formación (condiciones que favorecen el facilismo, la improvisación y la falta de rigurosidad).

Dificultades serias para consolidar un equipo docente competente (heterogeneidad de perfiles, intereses, niveles de compromiso, formación en investigación y horarios).

\begin{tabular}{|l|l|} 
& $\begin{array}{l}\text { certar horarios, de su disposición al } \\
\text { trabajo en equipo y para hacer apor- } \\
\text { tes al campo. }\end{array}$ \\
\hline $\begin{array}{l}\text { Dificultades serias para consolidar un } \\
\text { equipo docente por la enorme dispersión } \\
\text { al interior del equipo docente y la alta mo- } \\
\text { vilidad de los mismos. }\end{array}$ & $\begin{array}{l}\text { Reducir la movilidad de los docentes } \\
\text { de investigación y, de ser posible, su } \\
\text { dedicación privilegiada al campo. }\end{array}$ \\
\hline $\begin{array}{l}\text { La dispersión de docentes en los diferen- } \\
\text { tes semestres y grupos (muchos docentes } \\
\text { dispersos en diferentes niveles de forma- } \\
\text { ción) impide, no solo la consolidación de } \\
\text { conocimiento, de sus habilidades docentes } \\
\text { y de sus herramientas pedagógicas; los es- } \\
\text { tudiantes perciben rupturas dramáticas en } \\
\text { el proceso de su formación. }\end{array}$ & $\begin{array}{l}\text { Tener menos cantidad de docentes } \\
\text { do los diferentes niveles de for- } \\
\text { mación de modo que cada docente } \\
\text { pueda asumir los grupos de un mis- } \\
\text { mo nivel. }\end{array}$ \\
$\begin{array}{l}\text { Reubicar a los docentes en la se- } \\
\text { cuencia de formación de acuerdo a } \\
\text { su perfil y a las necesidades del cam- } \\
\text { po, favoreciendo su rendimiento. }\end{array}$ \\
\hline $\begin{array}{l}\text { La asignación de horas en nómina a los } \\
\text { docentes, apenas si permite el desarrollo } \\
\text { de los contenidos en el espacio de clase, } \\
\text { difícilmente la asesoría y nunca la revisión, } \\
\text { corrección y orientación de los productos. }\end{array}$ & $\begin{array}{l}\text { Aumentar el tiempo reconocido } \\
\text { en nómina para estos docentes de } \\
\text { modo que al menos tengan una hora } \\
\text { más de asesoría semanal y otra para } \\
\text { seguimiento a productos y avances } \\
\text { del ElS. }\end{array}$ \\
\hline
\end{tabular}

Aumentar el tiempo destinado al trabajo independiente, toda vez que se aumente el número de créditos reconocidos a los espacios de formación en investigación.

Seleccionar a los docentes de investigación a partir del perfil que como investigadores y formadores en investigación tengan, de su interés y compromiso con los lineamientos establecidos para el desarrollo del programa, de su posibilidad de concertar horarios, de su disposición al trabajo en equipo y para hacer aporTener menos cantidad de docentes centramación de modo que cada docente pueda asumir los grupos de un mis- 
La coordinación del campo implica una cantidad y visibilidad importante de actividades que Reconocer a los docentes del campo un convocan a toda la facultad, con un equipo de tiempo destinado a reuniones de campo docentes fracturado (diversidad de horarios, (discusión de programas, metodologías, intensidades horarias, compromiso con cam- formas de evaluación, diseño de planes pos alternos, motivación y dificultades de de homologación, suficiencia, ECAES y comunicación). - "lista de profesores que eje- preparatorios, socialización de pósteres, cutan (de manera más o menos ajustada) un construcción de comunidad académica). programa de clase.

Difícil articulación con coordinación de líneas.

Dificultades en el soporte logístico requerido para socialización de pósteres de investigación de los estudiantes.

Limitar la construcción de un EIS, con su correspondiente articulación a líneas, diseño de pósteres y socialización de informes finales, a los estudiantes de los ciclos de profundización y profesionalización.

El valor de este estudio radica en haber identificado los sectores de dificultad actual del programa, desde donde se plantean posibles soluciones que efectivamente se traducen en una propuesta de trasformación al plan de estudios, actualmente, en estudio por parte del Consejo de Facultad, que implica:

1. Una redistribución de contenidos y tiempos en ciclos básico y de profundización con el correspondiente ajuste en número de créditos académicos para los espacios de investigación.

2. Un período de transición de un semestre durante el cual los procesos de formación podrán unificarse en cada uno de los niveles que configuran la propuesta.

3. Unas estrategias de transición que implica concentrar el número de horas de clase/asesorías, de profesores responsables de las mismas, y ampliar el número de grupos en el nivel de lo que sería habilidades investigativas y diseño metodológico.

4. Entrada en plena vigencia en su forma final a partir del 2009-2. 


\section{Referencias}

Sastre, A. (2005). La formación en investigación: Una experiencia pedagógica. Manuscrito no publicado.

Sastre, A. (2006). Reporte de la evaluación realizada al programa de formación en competencias investigativas. Manuscrito no publicado.

Sastre, A. (2007). La investigación en la formación del profesional en Cultura Física, Deporte y Recreación. Documento del campo de la investigación. Bogotá: Facultad de Cultura Física, Deporte y Recreación - Universidad Santo Tomás. Manuscrito no publicado.

Sastre, A. (2006, 2007, 2008). Procedimientos para la construcción, asesoría y evaluación de ejercicios investigativos semestrales. Manuscrito no publicado.

USTA (2009). Recuperado desde [http://www.usta.edu.co]. 

